\title{
Nonhyperbolic homoclinic chaos
}

\author{
G. Cicogna ${ }^{(*)}$ and M. Santoprete \\ Dipartimento di Fisica, Università di Pisa, \\ Via Buonarroti 2, Ed. B \\ I-56127, Pisa, Italy
}

\begin{abstract}
.
Homoclinic chaos is usually examined with the hypothesis of hyperbolicity of the critical point. We consider here, following a (suitably adjusted) classical analytic method, the case of non-hyperbolic points and show that, under a Melnikov-type condition plus an additional assumption, the negatively and positively asymptotic sets persist under periodic perturbations, together with their infinitely many intersections on the Poincaré section. We also examine, by means of essentially the same procedure, the case of (heteroclinic) orbits tending to the infinity; this case includes in particular the classical Sitnikov 3-body problem.
\end{abstract}

PACS. no: $03.20,05.45$

Keywords: homoclinic chaos, nonhyperbolic critical point, Melnikov theory, Sitnikov problem.

To appear in Phys. Letters A

$\overline{(*)}$ E-mail : cicogna@difi.unipi.it 
It is well known that perturbing homoclinic (or heteroclinic) orbits of dynamical systems may lead to the phenomenon of transversal intersections of stable and unstable manifolds of the critical point, and that this is one of the routes for the appearance of chaos; it is also known that Melnikov method is a very efficient analytical criterion to determine the occurrence of the intersection of stable and unstable manifolds. It is impossible to give here even a partial account of the vast literature on this subject, and we will quote here only those papers [1-7] which are more strictly related to the present approach (for a more complete list of references, see also [8]).

It can be noticed, however, that almost the totality of the papers dealing with homoclinic chaos and Melnikov method considers only the case of hyperbolic critical points (some exceptions will be quoted below). We want to show here that a suitable extension of a classical and purely analytical method, used in [5] for the hyperbolic case, can also cover - under suitable hypotheses - the nonhyperbolic case.

For definiteness, even if more general situations could be considered, we will deal here only with planar systems originated from unperturbed Hamiltonians of the form

$$
H=\frac{1}{2} p^{2}+V(x)
$$

We point out that this method can (and will) be applied not only to the case of homoclinic orbits approaching nonhyperbolic points corresponding to some "superquadratic" unstable equilibrium points $x_{0} \in \mathbf{R}$ of the unperturbed potential $V(x)$, but also to the quite different case of (heteroclinic) orbits tending to $\pm \infty$ for $t \rightarrow \pm \infty$ respectively, appearing in the case of potentials having an equilibrium point at the infinity.

For the sake of concreteness, we shall provide the construction and the proof only for the case of superquadratic stationary points, but it will appear clear that the argument can be repeated equally well for the other one. For what concerns the first case, we refer also to [9], where however a completely different approach is used; the second situation includes in particular the classical Sitnikov restricted 3-body problem in celestial mechanics, which has been considered in detail by Moser [4], by means of the introduction of a singular change of coordinates (see also $[10,11]$ ).

More specifically, we will show that the negatively and positively asymptotic sets of the unstable equilibrium point persist even in the nonhyperbolic case and under periodic perturbation. We also obtain that the occurrence of an intersection of these sets on the Poincaré section (together with the chain of their infinitely many subsequent intersections) can be detected by a Melnikov criterion, plus an additional condition, which must be introduced in this case to compensate the lack of the "exponential dichotomy" peculiar of the hyperbolic case. 
1. We consider a Hamiltonian dynamical problem with Hamiltonian of the form (1), plus a smooth perturbation $g$ depending on one or more real (small) parameters $\epsilon$ :

$$
\begin{aligned}
& \dot{x}=p \\
& \dot{p}=-\frac{\mathrm{d} V}{\mathrm{~d} x}+g(\epsilon, x, \dot{x}, t) \quad \text { with } \quad g(0, x, \dot{x}, t)=0
\end{aligned}
$$

where the potential $V(x)$ of the unperturbed problem is assumed to be analytical and to have a "superquadratic" unstable equilibrium point at $x=x_{0}$ (say, $x_{0}=0$ ), which may be either a "cubic-like" stationary point, or a local maximum for $V(x)$ (in this case, clearly, $m<0$ in (3) below):

$$
V(0)=\frac{\mathrm{d} V(0)}{\mathrm{d} x}=\ldots \frac{\mathrm{d}^{\nu-1} V(0)}{\mathrm{d} x^{\nu-1}}=0 \quad ; \quad \frac{\mathrm{d}^{\nu} V(0)}{\mathrm{d} x^{\nu}}=m \neq 0 \quad \text { for some integer } \nu>2
$$

We assume that the unperturbed problem possesses a homoclinic orbit doubly asymptotic to $x_{0}=0$ :

$$
\chi=\chi(t) \quad \text { with } \quad \lim _{t \rightarrow \pm \infty} \chi(t)=0
$$

this happens, e.g., if $V(x)$ also admits at least a stable equilibrium point $x_{1}$ (e.g., with $x_{1}>x_{0}$ ), and there is another point $x_{2}>x_{1}$ such that $V\left(x_{2}\right)=0$ and $\mathrm{d} V(x) / \mathrm{d} x<0$ for $x_{0}<x<x_{1}, \mathrm{~d} V(x) / \mathrm{d} x>0$ for $x_{1}<x \leq x_{2}$.

A typical example of this situation is given by a double-well Duffing-type potential, modelling a great number of physical situations (this example will be considered explicitly at the end of this paragraph)

$$
V(x)=-\frac{1}{2} x^{4}+\frac{1}{2} x^{6}
$$

It is immediate to see that the unstable equilibrium point $x_{0}=0$ (or better, the point $x=0, p=0$ of the first-order problem (2), with a potential satisfying (3)) is not a hyperbolic point, indeed the linearized part of (2) (with $\epsilon=0$ ) is given by the nilpotent matrix

$$
\left(\begin{array}{ll}
0 & 1 \\
0 & 0
\end{array}\right)
$$

(cf. $[9,12]$, where existence and regularity of the invariant manifolds is investigated in a very general setting, see also [11]). A first consequence of this fact is that now the "exponential dichotomy" peculiar of the hyperbolic situation is no longer available; it is easy to see in fact that the homoclinic solution of the unperturbed problem vanishes in our case for $|t| \rightarrow \infty$ as

$$
\chi(t) \sim|t|^{-2 /(\nu-2)}
$$

Consider now the effect of the perturbation $g(\epsilon, x, \dot{x}, t)$ : here, we must assume first of all that

$$
g(\epsilon, 0,0, t)=0
$$

this ensures that $x_{0}=0$ is an equilibrium point also for the perturbed problem. The non-hyperbolicity of this point cannot ensure a priori the existence of stable and unstable manifolds; more precisely, the non-hyperbolicity has a more radical consequence: we cannot even properly speak of stable and unstable 
manifolds, indeed, the eigenvalues of the linearized problem are zero, and all the dynamics occurs actually on the center manifold (cf. [13]).

We now want to show that suitable assumptions on the perturbation $g$ may guarantee not only the existence of solutions approaching the equilibrium point $x_{0}=0$ for $t \rightarrow-\infty$ and for $t \rightarrow+\infty$, which we will denote respectively by

$$
x_{o}=x_{o}(t) \quad \text { and } \quad x_{i}=x_{i}(t)
$$

forming the "out-set" and the "in-set" - and playing the role of negatively and positively asymptotic sets of the hyperbolic case - but also the possible presence of transversal intersections on the Poincaré sections.

Let us write the problem (2) in the form (where $f=-\mathrm{d} V / \mathrm{d} x$ )

$$
\ddot{x}-f(x)=g(\epsilon, x, \dot{x}, t)
$$

and let us look for solutions $x(t)$ of the perturbed problem "near" the family of the homoclinic orbits $\chi\left(t-t_{0}\right)$ : we then put for convenience (see [5])

$$
x(t)=\chi\left(t-t_{0}\right)+z\left(t-t_{0}\right)
$$

Inserting into (8) (with the time shift $t-t_{0} \rightarrow t$ ), we obtain

$$
\ddot{z}-f^{\prime}[\chi(t)] z(t)=G\left(\epsilon, z, \dot{z}, t+t_{0}\right)
$$

where the r.h.s. is given by (we will shortly denote by $G=G\left(\epsilon, t, t_{0}\right)$ this quantity; notice that no approximation has been made, nor any term neglected)

$$
G=G\left(\epsilon, t, t_{0}\right) \equiv g\left(\epsilon, \chi(t)+z, \dot{\chi}(t)+\dot{z}, t+t_{0}\right)+f(\chi(t)+z)-f(\chi(t))-f^{\prime}[\chi(t)] z
$$

Consider the homogeneous equation obtained from (10): one solution of is clearly $\dot{\chi}(t)$, another independent solution $\psi(t)$ can be constructed with standard methods (see e.g. [14]) or by direct substitution: these two solutions have a different behaviour for $t \rightarrow \pm \infty$, precisely:

$$
\begin{aligned}
& \dot{\chi}(t) \sim|t|^{-\nu /(\nu-2)} \rightarrow 0 \\
& \psi(t) \sim|t|^{2(\nu-1) /(\nu-2)} \rightarrow \infty
\end{aligned}
$$

The general solution $z(t)$ (even if in implicit form, through the definition (11) of $G$ ) of the complete equation (10) can be written in the following integral form, with $A, B$ arbitrary constants and $t_{1}$ arbitrarily fixed:

$$
z(t)=A \dot{\chi}(t)+B \psi(t)-\dot{\chi}(t) \int_{t_{1}}^{t} \psi(s) G\left(\epsilon, s, t_{0}\right) \mathrm{d} s+\psi(t) \int_{t_{1}}^{t} \dot{\chi}(s) G\left(\epsilon, s, t_{0}\right) \mathrm{d} s
$$

Let us now look for solutions $z_{o}(t)$ (and resp. $z_{i}(t)$ ) of (13) with the property of vanishing for $t \rightarrow-\infty$ (resp. $t \rightarrow+\infty$ ); these will provide solutions

$$
x_{o, i}=\chi(t)+z_{o, i}(t)
$$

of (8) belonging, by definition, to the out-set (resp. the in-set) of $x_{0}=0$. We have then to require that

$$
\begin{aligned}
& \lim _{t \rightarrow \mp \infty}\left[\psi(t)\left(B+\int_{t_{1}}^{t} \dot{\chi}(s) G\left(\epsilon, s, t_{0}\right) \mathrm{d} s\right)\right]=0 \\
& \lim _{t \rightarrow \mp \infty}\left[\dot{\chi}(t)\left(A-\int_{t_{1}}^{t} \psi(s) G\left(\epsilon, s, t_{0}\right) \mathrm{d} s\right)\right]=0
\end{aligned}
$$


where the limits are to be evaluated at $-\infty$ when looking for the $x_{o}$ solutions, and resp. at $+\infty$ for the $x_{i}$ solutions. Notice that, in contrast with the hyperbolic case, we have to request here the vanishing of these quantities (not only their boundedness, as in [5]).

Now, it is an easy exercise to check, taking also into account the behaviour (12) of the two fundamental solutions $\dot{\chi}(t)$ and $\psi(t)$, that the above conditions (14) are satisfied simultaneously at $t=-\infty$ and $+\infty$ if the following (Melnikov-type condition)

$$
\int_{-\infty}^{+\infty} \dot{\chi}(t) G\left(\epsilon, t, t_{0}\right) \mathrm{d} t=0
$$

and the additional condition

$$
\lim _{t \rightarrow \mp \infty} \dot{\chi}(t) \int_{t_{1}}^{t} \psi(s) G\left(\epsilon, s, t_{0}\right) \mathrm{d} s=0
$$

are satisfied. Consider now the linearization of the problem (13) around the solution $z(t) \equiv 0, \epsilon=0$; in this way, condition (15) becomes a computable Melnikov condition

$$
M\left(\epsilon, t_{0}\right) \equiv \int_{-\infty}^{+\infty} \dot{\chi}(t) g\left(\epsilon, \chi(t), \dot{\chi}(t), t+t_{0}\right) \mathrm{d} t=0
$$

and the same is true for the other condition (16). Once these are satisfied, it is sufficient to apply the implicit-function theorem to be granted (see [5]) that there is a regular solution of (10), or equivalently of (13), close to the unperturbed solution $z \equiv 0$, i.e. a solution of (8) close to the homoclinics $\chi\left(t-t_{0}\right)$ and approaching $x_{0}=0$ for $t \rightarrow \pm \infty$.

Assume now also that

$$
\frac{\partial M}{\partial t_{0}} \neq 0
$$

the derivative being evaluated at the point $\epsilon, t_{0}$ where (17) is satisfied (this is of course "generically" true, see [15] for a careful discussion on non-transversal crossings) and that (16) also is satisfied (this may be easily fulfilled, see below for this point): there is then, thanks also to (18), a transversal intersection at $t=t_{0}$ on the Poincaré section of the out/in asymptotic sets of $x_{0}=0$. Using then standard arguments (not based on hyperbolicity), considering the case of perturbations $g$ which are periodic (or almost periodic) functions of the time, one immediately deduces that there is an infinite sequence of transversal intersections, leading to a situation similar to the usual chain of homoclinic intersections typical of the homoclinic chaos.

More exactly, we have now to emphasize that, in order to get a more complete description of the dynamical behaviour in this case, we cannot directly refer to the classical Birkhoff-Smale theorem on the equivalence with the Smale horseshoe dynamics, because the proofs of this equivalence are intrinsically based on hyperbolicity properties $[2-4,6,7]$. Some short comment about this point will be given at the end of this paper. Here, we can qualitatively say that the presence of infinitely many intersections indicates at least the presence of a quite complicated geometry in the behaviour of the dynamical flow, i.e. a sort of "nonhyperbolic homoclinic chaos" (cf. [9-13]).

We now give some conditions to ensure that (16) is satisfied. We assume here for instance that the perturbation $g$ can be written in the form

$$
g(\epsilon, x, \dot{x}, t)=\epsilon_{1} x^{n_{1}} \sin \omega t+\epsilon_{2} \dot{x}^{n_{2}}
$$


(notice, incidentally, that if the term with $\epsilon_{2}$ describes a damping and $n_{2}$ is even, then instead of $\dot{x}^{n_{2}}$ one must more correctly write $|\dot{x}| \dot{x}^{n_{2}-1}$, with $\epsilon_{2}>0$, of course), it is easy to check, recalling also (12), that if

$$
n_{1}>\nu-2 \quad \text { and } \quad n_{2}>\frac{2(\nu-2)}{\nu}
$$

then condition (16) is satisfied.

Considering e.g. the following explicit example, with $V$ given by (5),

$$
\ddot{x}=2 x^{3}-3 x^{5}+\epsilon_{1} x^{3} \sin \omega t+\epsilon_{2} \dot{x}^{3}
$$

there are two homoclinic orbits of the unperturbed Hamiltonian, given by

$$
\chi(t)=\frac{ \pm 1}{\sqrt{1+t^{2}}}
$$

conditions (20) are satisfied (here $\nu=4$ ) and the Melnikov condition (17) can be explicitly evaluated, giving transversal homoclinic intersections if

$$
\left|\frac{\epsilon_{1}}{\epsilon_{2}}\right|>\frac{3 \exp (\omega)}{32 \omega(1+\omega)}
$$

A numerical integration of this problem, performed along the same lines as in [6, Chapt.2] (see [8] for details), shows that, choosing e.g. $\omega=1, \epsilon_{2}=.05$, transversal intersections appear when $\epsilon_{1} \gtrsim .006$, in quite good agreement with the value $\epsilon_{1}=.00637$ given by $(22)$.

Summarizing, we can state the above results in following form:

Theorem. Consider a planar dynamical system of the form (2) where the potential $V(x)$ admits a superquadratic unstable equilibrium in some point, say $x_{0}=0$, producing then a nonhyperbolic unstable equilibrium point for the unperturbed system (given by $\epsilon=0$ ). Assume that the unperturbed system admits a homoclinic orbit $\chi(t)$ doubly asymptotic to $x_{0}$. Assume for simplicity the perturbation $g$ of the form (19) with the conditions (20) satisfied. If in addition Melnikov conditions (17) and (18) are satisfied, then the perturbed problem admits a negatively and positively asymptotic sets of the unstable equilibrium point, which admit an infinite sequence of homoclinic intersections on the Poincaré section, giving rise to a (nonhyperbolic homoclinic) chaotic dynamical flow.

2. Instead of discussing some of the possible extensions of the above results to more general dynamical systems or to problems in greater dimension, we prefer here to examine a quite different situation which in fact can be discussed with a similar procedure. Let us consider a problem with Hamiltonian (1), where now the potential is such that

$$
V(x)<0 \quad, \quad \forall x \in \mathbf{R} \quad \text { with } \quad \lim _{x \rightarrow \pm \infty} V(x)=0
$$

and there is only a stationary point $x_{0}$ (a minimum) for $V(x)$ :

$$
\frac{\mathrm{d} V\left(x_{0}\right)}{\mathrm{d} x}=0
$$


There is then a (heteroclinic) orbit approaching $-\infty$ and $+\infty$ :

$$
\chi=\chi(t) \quad \text { with } \quad \lim _{t \rightarrow \mp \infty} \chi(t)=\mp \infty
$$

A well known example is given by the classical Sitnikov restricted 3-body problem in celestial mechanics, which - at the limit of zero eccentricity - is described by the potential [4]

$$
V(x)=\frac{-1}{\sqrt{x^{2}+\frac{1}{4}}}
$$

More in general, we can consider a potential such that

$$
V(x) \sim \frac{1}{|x|^{\mu}} \quad \text { for } \quad|x| \rightarrow \infty \quad \text { with } \quad \mu>0, \text { real }
$$

Clearly, these problems are, per se, nonhyperbolic, the equilibrium point being at the infinity; actually, Moser [4] examined the above problem (25), in the presence of the periodic perturbation produced by nonzero eccentricity, using the McGehee [16] singular coordinate transformation defined by $x=2 / y^{2}$ and $\mathrm{d} t=\left(4 / y^{3}\right) \mathrm{d} s$, which in fact transforms the problem into a hyperbolic one near the point $y=0$, and he was able to prove the existence of Smale horseshoe dynamics (see also $[10,11]$ ).

Our above procedure can be equally well used in this new case, i.e. for problems with potential of the form $(23,27)$ : indeed, writing the problem as in (8), we can again introduce the variation equation (10-11). In this case, one finds that two independent solutions of the homogeneous part of (10) have the following behaviour, for $|t| \rightarrow \infty$

$$
\begin{aligned}
& \dot{\chi}(t) \sim|t|^{-\mu /(2+\mu)} \rightarrow 0 \\
& \psi(t) \sim|t|^{(2+2 \mu) /(2+\mu)} \rightarrow \infty
\end{aligned}
$$

This allows us to repeat exactly the same arguments as in part 1. In particular, assuming e.g. a nondissipative periodic perturbation of the form, for large $|x|$,

$$
g(\epsilon, x, \dot{x}, t) \sim \epsilon x^{-n} \sin \omega t
$$

it is easy to show that the existence of out/in sets is granted if

$$
n>2+\mu
$$

and to show also that the Melnikov function (17) possesses infinitely many transversal zeroes. Notice that the Sitnikov 3-body problem above mentioned falls into this situation, indeed - at the first order in the eccentricity $\epsilon$ - the perturbation is given by [4]

$$
g=\epsilon \frac{-3 x}{4\left(x^{2}+\frac{1}{4}\right)^{5 / 2}} \cos t
$$

As already remarked in part 1, the standard Birkhoff-Smale theorem cannot be directly used for this nonhyperbolic case. Here, however, at least for the case of Sitnikov problem, we can refer to the arguments used in [10] (see especially the Appendix of [10], and also [13]), to obtain an equivalence to a "nonhyperbolic 
horseshoe", where contracting and expanding actions are not exponential but "polynomial" in time. Alternatively, in the general case, one may possibly resort to the method of "blowing-up", devised to investigate the properties of nonhyperbolic singularities by means of suitable changes of coordinates [17,18], but presumably a full and general treatment of nonhyperbolic horseshoes is still open.

\section{Acknowledgments.}

We are grateful to prof.s D. Bambusi, U. Bessi, L. Galgani, and J. Mallet-Paret for useful suggestions and bibliografical indications.

\section{References}

[1] V.K. Melnikov, Trans. Moscow Math. Soc. 12 (1963) 1

[2] S. Smale, Bull. Amer. Math. Soc. 73 (1967) 747

[3] Z. Nitecki, Differentiable dynamics (MIT Press, Cambridge, Mass. 1971)

[4] J. Moser, Stable and random motions in dynamical systems (Princeton Univ. Press, Princeton 1973)

[5] S.-N. Chow, J.K. Hale and J. Mallet-Paret, J. Diff. Eq. 37 (1980) 351

[6] J. Guckenheimer and P.J. Holmes, Nonlinear oscillations, dynamical systems and bifurcations of vector fields (Springer, Berlin 1983)

[7] S. Wiggins, Global bifurcations and chaos (Springer, Berlin 1989)

[8] M. Santoprete, Thesis, Dept. of Physics, Univ. of Pisa

[9] J. Casasayas, E. Fontich and A. Nunes, Nonlinear Diff. Eq. Appl. 4 (1997) 201

[10] H. Dankowicz and P. Holmes, J. Diff. Eq. 116 (1995) 468

[11] C. Robinson, Contemp. Math. 198 (1996) 45

[12] J. Casasayas, E. Fontich and A. Nunes, Nonlinearity 5 (1992) 1193

[13] Xiao-Biao Lin, Dynamics Reported 5 (1996) 99

[14] E.A. Coddington and N. Levinson, Theory of ordinary differential equations (McGraw-Hill, New York 1955)

[15] V. Rayskin, preprint 1998, Dept. of Math., Texas Univ., Austin.

[16] R. McGehee, J. Diff. Eq. 14 (1973) 70

[17] F. Dumortier, J. Diff. Eq. 23 (1977) 53

[18] M. Brunella and M. Miari, J. Diff. Eq. 85 (1990) 338 